\title{
Immunotherapies for autoimmune diseases
}

\author{
Autoimmune diseases should benefit from treatments that piggyback on the successes of cancer \\ immunotherapies.
}

W hether by transplantation of tumour-specific engineered T cells, or by the administration of drugs that block immune checkpoints, activating or boosting the immune system can be highly efficacious for treating cancers, at least for those patients who respond well to such immunomodulation. Hundreds of past and ongoing clinical trials have tested, and are testing, the safety and efficacy of these immunotherapies, as well as of combinations of immunotherapies and traditional anticancer therapies involving radiation or cytotoxic drugs, for a wide range of cancers.

Immunotherapies have long been used to treat allergies, to reduce the rejection of transplanted organs and to dampen autoimmunity (for instance, inhibitors of tumour necrosis factor alpha, TNF- $\alpha$, are routinely used to reduce inflammation in diseases such as rheumatoid arthritis, inflammatory bowel disease and psoriasis). Yet, the latest cancer immunotherapies target a narrower set of immune pathways (as is the case for T-cell-specific immune checkpoint inhibitors, such as programmedcell-death protein $1, \mathrm{PD}-1$, and cytotoxic T-lymphocyte-associated protein 4 , CTLA-4) or elicit responses only in the presence of tumour-associated antigens (TAAs). This new generation of targeted immunotherapies is now being adapted for treating autoimmune diseases.

A Comment by Dominic Boardman and Megan Levings in this issue discusses the advantages and limitations of adapting cancer-immunotherapy drugs (including cytokines, biologics and engineered cells) for the treatment of autoimmune diseases. Although immunotherapies for treating cancer and those for treating autoimmunity seek opposite effects on the immune system - in cancer, to prime or enhance immune responses against tumours; in autoimmunity, to promote immune suppression and curb inflammation - both types of immunotherapy can activate (in cancer) or inhibit (in autoimmunity) the same immune pathways. Indeed, chimaeric antigen receptor (CAR)-T cells can target autoantigens to suppress autoimmunity (Fig. 1), and immune checkpoints may be leveraged to dampen the activity of the pathogenic $\mathrm{T}$ cells that drive autoimmunity.

In fact, a study by Mingnan Chen and

colleagues also included in this issue shows that specifically depleting PD-1-expressing lymphocytes with an immunotoxin ameliorates autoimmunity in mice. The immunotoxin complex consists of a single-chain variable fragment that binds to PD-1, a Pseudomonas exotoxin that selectively targets and induces the killing of PD-1-expressing T cells, and an albuminbinding domain, to extend the half-life of the complex in circulation and to improve its pharmacokinetics. Administration of the immunotoxin delayed disease onset when administered to mouse models of diabetes, and ameliorated symptoms in mice paralysed by experimental autoimmune encephalomyelitis. Importantly, the normal immune function of the treated mice was largely preserved after treatment (both treated and control mice immunized against a T-cell-independent antigen displayed similar levels of antibodies).

Boosting or suppressing the immune system to treat disease is a delicate matter, as it can lead to adverse effects, such as fungal infections and lymphomas (in patients treated with TNF inhibitors) as well as the acute triggering of autoimmunity or cytokine-release syndrome (in patients treated with immune checkpoint inhibitors or CAR-T cells). Therefore, patients being treated with immunotherapies have to be closely observed, typically via the monitoring of immune processes and of biomarkers associated with immune activation. In this respect, Andrew Adams, Gabriel Kwong and colleagues report, in another study in this issue, a urinary nanosensor of the activity of granzyme $\mathrm{B}$ - a factor released by activated cytotoxic $\mathrm{T}$ cells - for the early diagnosis of the acute rejection of transplanted skin allografts in mice. The ability to indirectly detect activated $\mathrm{T}$ cells non-invasively might eventually be used to monitor patients treated with immunotherapies.

The race to extend the application of immunotherapies to as many cancer types as possible will run in parallel with efforts toward repurposing some of the therapies used for treating autoimmunity. For the

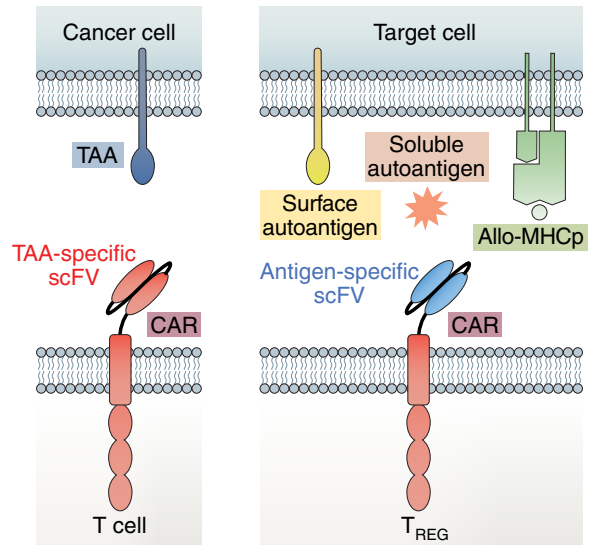

Fig. 1 | CAR-T cells can target autoantigens to suppress autoimmunity. Similar to CAR-T cells targeting TAAs expressed on the surface of cancer cells, autoimmunity can be suppressed via CAR-T cells targeting autoantigens, soluble autoantigens or allogeneic major-histocompatibility-peptide complexes (Allo-MHCps; expressed by donor cells). $\mathrm{ScFv}$, single-chain variable fragment; $\mathrm{T}_{\mathrm{REG}}$, regulatory $\mathrm{T}$ cell. Figure reproduced from the Comment by Boardman and Levings, Springer Nature Ltd.

latter, the drugs will have to be modified to block, rather than activate, immune factors, and the antigen receptors on engineered T cells targeting tumour peptides will have to be replaced with constructs targeting autoimmunity-relevant factors. However, the targeted immune pathways and cellengineering techniques relevant to cancer immunotherapy could be readily adapted for autoimmunity. Still, at least for checkpoint inhibitors, expanding the applications of immunotherapies from one cancer type to another should be easier than repurposing them for autoimmunity. As Boardman and Levings suggest in their Comment, "there is clearly a strong rationale for further expanding the opportunities for crossfertilisation of ideas and approaches between cancer immunology and autoimmunity, so that further synergies between the two fields can accelerate the development of effective immunotherapies."

Published online: 5 April 2019

https://doi.org/10.1038/s41551-019-0394-3 